\title{
TRANSFERÊNCIA DE OXIGÊNIO E TEMPO DE CIRCULAÇÃO EM BIORREATORES AIRLIFT SPLIT COM DIFERENTES GEOMETRIAS DA REGIÃO DE MISTURA
}

\author{
A. C. MEIRA ${ }^{1}$, M. N. ESPERANÇA ${ }^{1}$ e A. C. BADINO ${ }^{1}$ \\ ${ }^{1}$ Universidade Federal de São Carlos, Departamento de Engenharia Química \\ E-mail para contato: alanacm8@gmail.com
}

\begin{abstract}
RESUMO - O presente trabalho avaliou a influência da geometria da região de mistura no desempenho de biorreatores airlift split. Para isso, foram analisados os parâmetros transferência de oxigênio e tempo de circulação para diferentes geometrias, variando-se o ângulo e a fração volumétrica de líquido contida na região de mistura e utilizando biorreator de $10 \mathrm{~L}$ de volume útil e água destilada como fluido. Verificou-se que a geometria da região de mistura possui influência significativa apenas na transferência de oxigênio.
\end{abstract}

\section{INTRODUÇÃO}

Os biorreatores não convencionais pneumáticos tipo coluna de bolhas e tipo airlift têm merecido destaque no campo da biotecnologia devido a características como baixo consumo de energia, capacidade relativamente alta de transferir oxigênio para o meio de cultura, ausência de partes móveis e facilidade de construção, operação e aumento de escala. Por isso, esses biorreatores têm se mostrado uma alternativa promissora ao biorreator convencional tipo tanque agitado e aerado (THOMASI 2010).

O desempenho de biorreatores pneumáticos tipo airlift é avaliado através de parâmetros como o coeficiente volumétrico de transferência de oxigênio $\left(\mathrm{k}_{\mathrm{L}} \mathrm{a}\right)$ e o tempo de circulação $\left(\mathrm{t}_{\mathrm{c}}\right)$. Além de depender da vazão de alimentação do ar $\left(\phi_{\mathrm{AR}}\right)$ e do fluido presente no equipamento, estes parâmetros são influenciados pela geometria do biorreator, tendo como destaque a região de mistura, que proporciona o desprendimento de parte do gás e, consequentemente, a circulação do líquido. Assim, o presente estudo avaliou a influência da geometria da região de mistura em biorreatores airlift de circulação interna do tipo split, variando-se o ângulo $(\alpha)$ e a fração volumétrica de líquido contida na região de mistura $\left(\mathrm{FV}_{\mathrm{L}, \mathrm{RM}}\right)$.

\section{MATERIAIS E MÉTODOS}

\subsection{Biorreatores}

Os experimentos foram conduzidos em biorreatores airlift split de $10 \mathrm{~L}$ de volume útil, confeccionados em acrílico. Estes equipamentos eram compostos por um corpo principal, que possui $160 \mathrm{~mm}$ de diâmetro, associado a um corpo cônico (Figura 1) e equipados com um aspersor do tipo "espinha de peixe", com furos de $0,5 \mathrm{~mm}$ de diâmetro, espaçados de $5 \mathrm{a} 5 \mathrm{~mm}$. A avaliação do efeito da geometria da região de mistura do biorreator airlift foi realizada utilizando-se diferentes ângulos e frações de líquido da região de mistura. A fração volumétrica 
de líquido na região de mistura é definida como a razão entre o volume de líquido contido na região de mistura para o sistema não aerado $\left(\mathrm{V}_{\mathrm{L}, \mathrm{RM}}\right)$ e o volume de líquido total do biorreator $\left(\mathrm{V}_{\mathrm{L}}\right)$ (ESPERANÇA, 2014), conforme a Equação 1:

$F V_{L, R M}=\frac{V_{L, R M}}{V_{L}}$

Figura 1 - Esquema de um biorreator airlift split com região de mistura expandida.

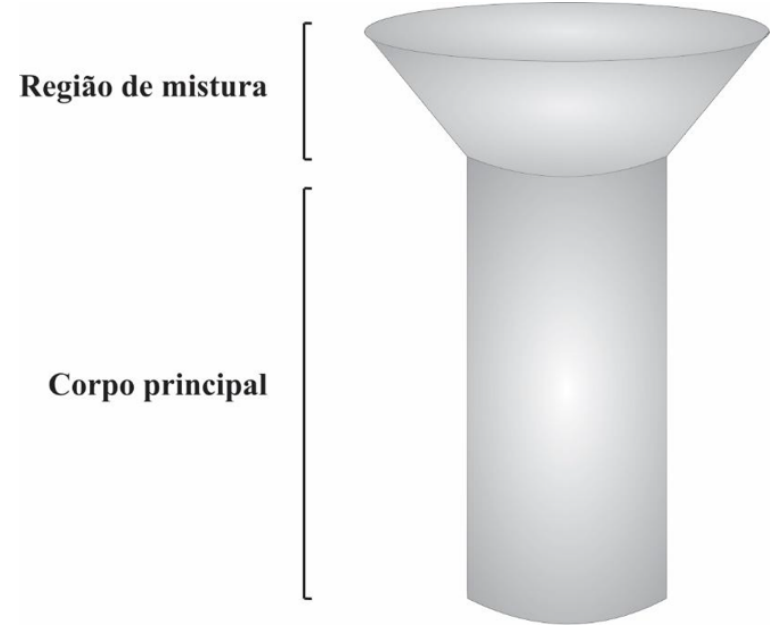

(Acervo pessoal)

Água destilada ( $\left.\mu=8,7.10^{-4} \mathrm{~Pa} . \mathrm{s}\right)$ foi utilizada como fluido modelo nos ensaios.

\subsection{Condições Experimentais}

Para avaliar a influência da geometria da região de mistura ( $\alpha$, ângulo; e $F V_{L, R M}$, fração volumétrica de líquido na região de mistura) e da vazão específica de ar $\left(\phi_{\mathrm{AR}}\right)$ sobre os parâmetros de desempenho, foi empregada a metodologia de delineamento composto central rotacional (DCCR) (RODRIGUES e IEMMA, 2005), cujos valores reais e codificados das variáveis independentes são apresentados na Tabela 1 .

Tabela 1 - Valores reais e codificados das variáveis independentes.

\begin{tabular}{lcccccc}
\hline & & \multicolumn{5}{c}{ Níveis } \\
\cline { 3 - 7 } \multicolumn{1}{c}{ Variável } & Código & $-1,68$ & -1 & 0 & 1 & 1,68 \\
\hline$\alpha\left(^{\circ}\right)$ & $\mathrm{x}_{1}$ & 30 & 42 & 60 & 78 & 90 \\
$\mathrm{FV}_{\mathrm{L}, \mathrm{RM}}(-)$ & $\mathrm{x}_{2}$ & 0,10 & 0,14 & 0,20 & 0,26 & 0,30 \\
$\Phi_{\mathrm{AR}}(\mathrm{vvm})$ & $\mathrm{x}_{3}$ & 1,0 & 1,8 & 3,0 & 4,2 & 5,0 \\
\hline
\end{tabular}

Através de um software estatístico (STATISTICA v. 7.0) os resultados foram analisados, avaliando-se os efeitos das variáveis independentes, além de se determinar os fatores estatisticamente significativos a um nível de significância de 10\% (Intervalo de confiança de $90 \%)$. 


\subsection{Determinação do coeficiente volumétrico de transferência de oxigênio}

O coeficiente volumétrico de transferência de oxigênio $\left(\mathrm{k}_{\mathrm{L}} \mathrm{a}\right)$ foi determinado através do método dinâmico (CHISTI, 1989), considerando-se o atraso da resposta do eletrodo de primeira ordem (Aiba, Humphrey e Millis, 1973). Este método consiste em utilizar um eletrodo para medir a concentração de oxigênio dissolvido no meio líquido $\left(C_{e}\right)$, promovendo-se uma alteração nesta concentração a partir de um ensaio degrau $\left(\mathrm{N}_{2} \rightarrow\right.$ Ar). Através de um sistema de aquisição de dados em tempo real, obteve-se um perfil de $C_{e}$ em função do tempo $(t)$.

$\mathrm{O}$ valor de $\mathrm{k}_{\mathrm{L}} \mathrm{a}$ foi obtido ajustando-se a Equação (2) aos dados experimentais de concentração de oxigênio dissolvido em função do tempo através de regressão não-linear, conhecido o valor de $\mathrm{k}_{\mathrm{e}}$.

$C_{e}=C_{e 0} \cdot e^{-k_{e} \cdot\left(t-t_{0}\right)}+C_{e S} \cdot\left(1-e^{-k_{e} \cdot\left(t-t_{0}\right)}\right)+\frac{k_{e} \cdot\left(C_{e S}-C_{O_{20}}\right)}{k_{e}-k_{L} a} \cdot\left(e^{-k_{e} \cdot\left(t-t_{0}\right)}-e^{-k_{L} a \cdot\left(t-t_{0}\right)}\right)(2)$

sendo:

$\mathrm{C}_{\mathrm{e} 0}$ : concentração de oxigênio dissolvido medida pelo eletrodo na condição inicial.

$\mathrm{C}_{\mathrm{eS}}$ : concentração de oxigênio dissolvido medida pelo eletrodo na condição de saturação.

\subsection{Determinação do tempo de circulação}

O tempo de circulação $\left(\mathrm{t}_{\mathrm{C}}\right)$ foi determinado através do método da esfera (VASCONCELOS et al., 2003), no qual uma partícula de densidade semelhante à do fluido utilizado é inserida no biorreator em funcionamento. Com o auxílio de uma câmera fotográfica, a região da base do biorreator foi filmada durante 2 minutos, permitindo assim a determinação do número de voltas completas (n) da esfera no biorreator para um determinado intervalo de tempo $(\Delta \mathrm{t})$. O tempo de circulação é dado pela Equação 3.

$t_{C}=\frac{\Delta t}{n}$

\section{RESULTADOS E DISCUSSÃO}

Os resultados de $\mathrm{k}_{\mathrm{L}} \mathrm{a}$ e de tc para biorreatores airlift split operando com água destilada a $32^{\circ} \mathrm{C}$ são apresentados na Tabela 2.

Observa-se pela Tabela 2, que ocorreu uma variação de $\mathrm{k}_{\mathrm{L}}$ a de $0,0163 \mathrm{~s}^{-1}$ a $0,0633 \mathrm{~s}^{-1}$. Este intervalo de variação foi semelhante ao obtido por Esperança (2014) em biorreator airlift de dutos concêntricos de $10 \mathrm{~L}$, operando com água destilada, que variaram de $0,0208 \mathrm{~s}^{-1} \mathrm{a}$ $0,0683 \mathrm{~s}^{-1}$. Além disso, foi possível observar que a faixa de tempo de circulação variou de 3,43 s a 5,50 s. O intervalo foi semelhante ao encontrado por Cerri (2009) em biorreator airlift de 10 L, operando com água destilada, que variou de 2,54 s a 4,53 s.

A análise de variância (ANOVA) com intervalo de confiança de $90 \%$ resultou no modelo refinado descrito pela Equação 4 para o $\mathrm{k}_{\mathrm{L}} \mathrm{a}$ e na Equação 5 para o $\mathrm{t}_{\mathrm{C}}$. Para ambos parâmetros de desempenho, o valor de $\mathrm{F}$ calculado foi pelo menos 5,78 vezes maior do que o tabelado (Tabela 3), validando os modelos para serem utilizados na obtenção de superfícies de resposta. 
Tabela 2 - Matriz do DCCR com resultados de $\mathrm{k}_{\mathrm{L}} \mathrm{a}$ e $\mathrm{t}_{\mathrm{C}}$ para biorreatores airlift $\mathrm{split}$ operando com água destilada.

\begin{tabular}{|c|c|c|c|c|c|}
\hline Ensaios & $\mathbf{x}_{\mathbf{1}}$ & $\mathbf{x}_{\mathbf{2}}$ & $\mathbf{x}_{\mathbf{3}}$ & $\mathbf{k}_{\mathbf{L}} \mathbf{a}\left(\mathbf{s}^{-\mathbf{1}}\right)$ & $\mathbf{t}_{\mathbf{C}} \mathbf{( s )}$ \\
\hline $\mathbf{1}$ & -1 & -1 & -1 & 0,0360 & 4,20 \\
\hline $\mathbf{2}$ & 1 & -1 & -1 & 0,0304 & 4,23 \\
\hline $\mathbf{3}$ & -1 & 1 & -1 & 0,0258 & 4,14 \\
\hline $\mathbf{4}$ & 1 & 1 & -1 & 0,0306 & 4,58 \\
\hline $\mathbf{5}$ & -1 & -1 & 1 & 0,0633 & 3,81 \\
\hline $\mathbf{6}$ & 1 & -1 & 1 & 0,0472 & 3,87 \\
\hline $\mathbf{7}$ & -1 & 1 & 1 & 0,0466 & 3,43 \\
\hline $\mathbf{8}$ & 1 & 1 & 1 & 0,0561 & 3,84 \\
\hline $\mathbf{9}$ & $-1,68$ & 0 & 0 & 0,0339 & 4,08 \\
\hline $\mathbf{1 0}$ & 1,68 & 0 & 0 & 0,0235 & 3,77 \\
\hline $\mathbf{1 1}$ & 0 & $-1,68$ & 0 & 0,0438 & 3,85 \\
\hline $\mathbf{1 2}$ & 0 & 1,68 & 0 & 0,0359 & 4,17 \\
\hline $\mathbf{1 3}$ & 0 & 0 & $-1,68$ & 0,0163 & 5,50 \\
\hline $\mathbf{1 4}$ & 0 & 0 & 1,68 & 0,0424 & 3,52 \\
\hline $\mathbf{1 5}$ & 0 & 0 & 0 & 0,0345 & 4,15 \\
\hline $\mathbf{1 6}$ & 0 & 0 & 0 & 0,0345 & 4,04 \\
\hline $\mathbf{1 7}$ & 0 & 0 & 0 & 0,0331 & 3,88 \\
\hline
\end{tabular}

$k_{L} a=0,034+0,004 \cdot x_{2}^{2}+0,010 \cdot x_{3}+0,005 \cdot x_{1} \cdot x_{2}$

$t_{C}=3,931-0,405 \cdot x_{3}+0,164 \cdot x_{3}^{2}$

Tabela 3 - Análise de variância para os modelos que descrevem $\mathrm{k}_{\mathrm{L}} \mathrm{a}$ e $\mathrm{t}_{\mathrm{C}}$ em biorreator airlift split.

\begin{tabular}{|c|c|c|c|c|}
\hline & $\mathbf{R}^{2}$ & $\mathbf{F}_{\text {tab }}$ & $\mathbf{F}_{\text {calc }}$ & $\mathbf{F}_{\text {tab }} / \mathbf{F}_{\text {calc }}$ \\
\hline $\mathbf{k}_{\mathbf{L}} \mathbf{a}$ & 0,77 & 2,56 & 14,8 & 5,78 \\
\hline $\mathbf{t}_{\mathbf{C}}$ & 0,76 & 2,73 & 21,9 & 8,02 \\
\hline
\end{tabular}

Analisando-se a Equação 4, verifica-se a influência da geometria da região de mistura e da vazão específica do ar na determinação do $\mathrm{k}_{\mathrm{L}} \mathrm{a}$. Já em relação ao $\mathrm{t}_{\mathrm{C}}$, ao analisar a Equação 5 , verifica-se apenas a influência da vazão específica do ar. A Figura 2 apresenta uma comparação dos valores de tempo de circulação experimentais e previstos pela Equação 5, notando-se uma distribuição razoável dos pontos.

Para a melhor visualização do impacto das variáveis independentes sobre o $\mathrm{k}_{\mathrm{L}} \mathrm{a}$, foram geradas superfícies de resposta para esse parâmetro, em função do ângulo de abertura e da fração volumétrica de líquido na região de mistura, fixando-se três vazões diferentes, mostradas na Figura 3. 


\section{Congresso Brasileiro de Engenharia Química \\ em Iniciação Científica \\ UFSCar - São Carlos - SP 16 a 19 de Julho de 2017}

Figura 2 - Valores previstos em função dos valores observados de tempo de circulação para biorreator airlift split.

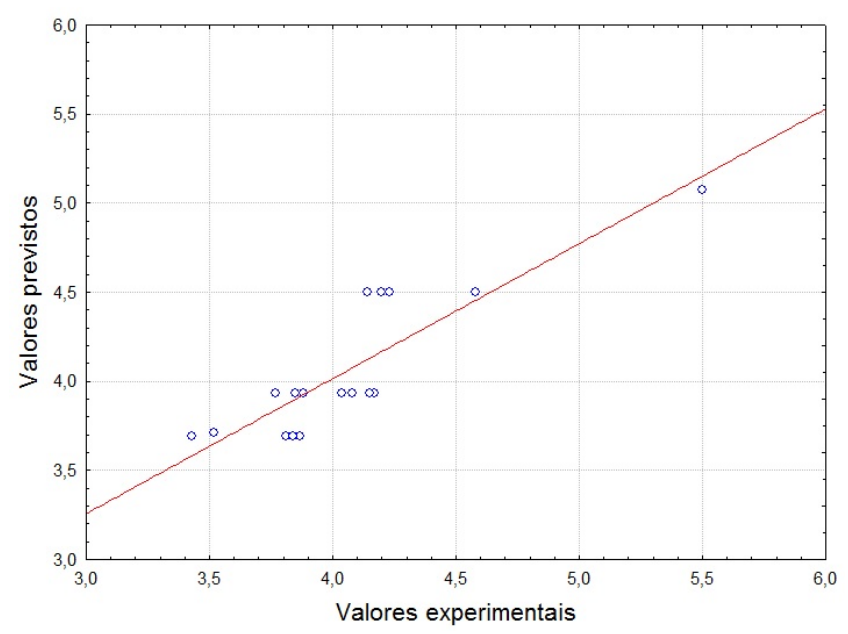

Figura 3 - Gráfico de contorno do coeficiente volumétrico de transferência de oxigênio em função do ângulo de abertura e da fração de líquido na região de mistura: (a) $\mathrm{x}_{3}=-1,68$

$$
\left(\phi_{\mathrm{AR}}=1,0 \mathrm{vvm}\right) ;(b) \mathrm{x}_{3}=0\left(\phi_{\mathrm{AR}}=3,0 \mathrm{vvm}\right) ;(\mathrm{c}) \mathrm{x}_{3}=1,68\left(\phi_{\mathrm{AR}}=5,0 \mathrm{vvm}\right) \text {. }
$$

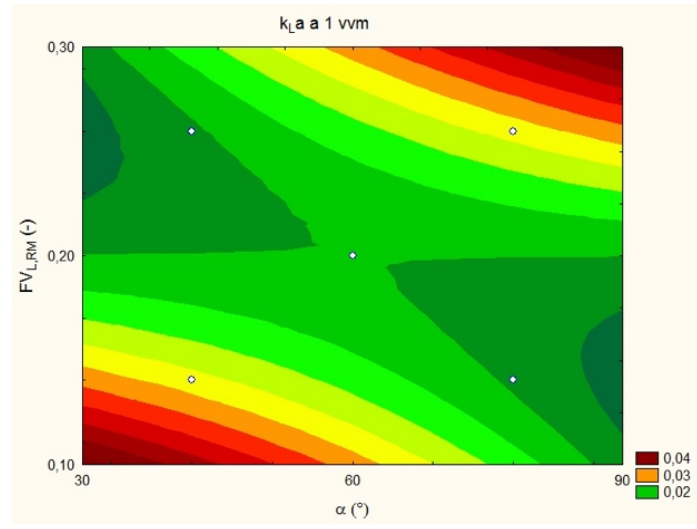

(a)

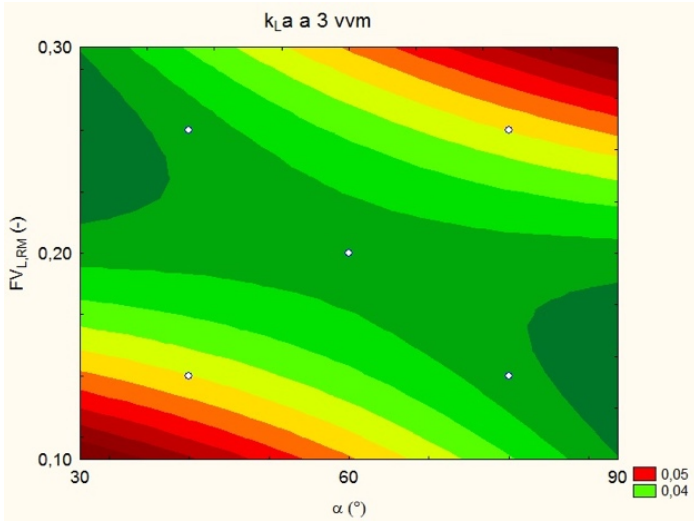

(b)

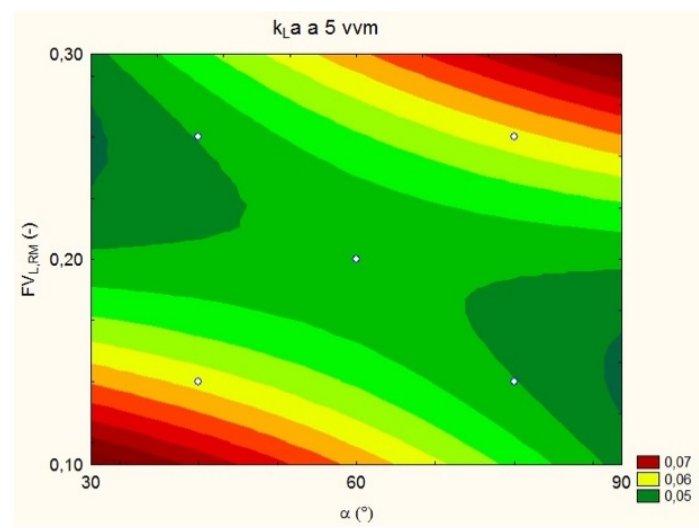

(c) 
Analisando-se a Figura 3, verificou-se que para ângulos de abertura medianos $\left(60^{\circ}\right) \mathrm{e}$ frações volumétricas de líquido na região de mistura de aproximadamente 0,20 , foram obtidos baixos valores de $\mathrm{k}_{\mathrm{L}} \mathrm{a}$, enquanto que para os maiores ângulo e fração de líquido $\left(\alpha=90^{\circ} \mathrm{e}\right.$ $\left.\mathrm{FV}_{\mathrm{L}, \mathrm{RM}}=0,30\right)$ ou para os menores ângulo e fração de líquido $\left(\alpha=30^{\circ}\right.$ e $\left.\mathrm{FV}_{\mathrm{L}, \mathrm{RM}}=0,10\right)$, foram observados maiores valores de $\mathrm{k}_{\mathrm{L}} \mathrm{a}$.

\section{CONCLUSÕES}

O presente trabalho avaliou a influência da geometria da região de mistura em biorreatores airlift split. Para isso, foram analisadas as variações dos parâmetros $\mathrm{k}_{\mathrm{L}} \mathrm{a}$ e $\mathrm{t}_{\mathrm{C}}$ quanto à $\phi_{\mathrm{AR}}$, ao ângulo $(\alpha)$ e à fração volumétrica de líquido contida na região de mistura $\left(\mathrm{FV} \mathrm{L}_{\mathrm{L}, \mathrm{RM}}\right)$. Verificou-se influência da geometria apenas na transferência de oxigênio, a qual também é influenciada pela $\phi_{\mathrm{AR}}$. Neste caso, as melhores condições de transferência de oxigênio foram observadas para biorreatores com ângulos de $90^{\circ}$ e fração de líquido de $30 \%$ e ângulos de $30^{\circ}$ e fração de líquido de $10 \%$. Em relação ao tempo de circulação, verificou-se influência apenas da $\phi_{\text {AR. }}$.

\section{REFERÊNCIAS BIBLIOGRÁFICAS}

AIBA, S.; HUMPHREY, A. E.; MILLIS, N. F. Biochemical Engineering. 2nd. Tokio: University of Tokio Press, 1973.

CERRI, M. O. Hidrodinâmica e transferência de oxigênio em três biorreatores airlift de circulação interna geometricamente semelhantes. 2009. Doutorado em Engenharia Química. Departamento de Engenharia Química, Universidade Federal de São Carlos.

CHISTI, Y. Airlift bioreactors. Belfast, Northern Ireland: Elsevier Science Publishers Ltd., 1989.

ESPERANÇA, M. N. Influência de aspectos geométricos na hidrodinâmica e transferência de oxigênio de biorreatores airlift de circulação interna.2014. Mestrado em Engenharia Química. Departamento de Engenharia Química, Universidade Federal de São Carlos.

RODRIGUES, M. I.; IEMMA, A. F. Planejamento de experimentos e otimização de processos: Uma estratégia sequencial de planejamentos. Casa do Pão Editora, 2005.

THOMASI, S. S. Avaliação de parâmetros de desempenho de três modelos de biorreatores pneumáticos de bancada. 2010. Mestrado em Engenharia Química. Departamento de Engenharia Química, Universidade Federal de São Carlos.

VASCONCELOS, J. M. T.; RODRIGUES, J. M. L.; ORVALHO, S. C. P.; ALVES, S. S.; MENDES, R. L.; REIS, A. Effect of contaminants on mass transfer coefficients in bubble column and airlift contactors. Chemical Engineering Science, v. 58, n. 8, p. 1431-1440, 2003. 